\title{
Educação em saúde bucal na visão de acadêmicos de Odontologia
}

\section{Perception of oral health education by dental students}

\author{
Karina Tonini dos Santos ${ }^{1}$, Antonio Carlos Pacheco Filho², Cléa Adas Saliba Garbin²
}

\section{RESUMO}

Objetivo: Analisar a visão dos acadêmicos do último ano do Curso de Odontologia sobre Educação em Saúde Bucal. Materiais e Métodos: A pesquisa, quanti-qualitativa, aborda aspectos relacionados com o entendimento dos acadêmicos sobre Educação em Saúde Bucal, o valor que atribuem a essa prática no âmbito escolar, bem como a importância da participação de atividades em outros cenários de práticas na formação. Resultados: Apesar de os acadêmicos reconhecerem a importância dos Programas de Educação em Saúde Bucal no âmbito escolar e também de sua participação nessas atividades, sua percepção sobre o que é "Educação em Saúde Bucal" é reducionista e centrada na doença. Conclusão: Sabe-se que tais fatos encontrados são reflexos e consequências de todo um contexto estabelecido na formação universitária. Tornase necessário, então, um profundo repensar de prática por parte de todos os formadores desses profissionais, para que possam desenvolver metodologias que privilegiem a Educação em Saúde em suas práticas de ensino. Descritores: Educação em saúde. Odontologia. Saúde bucal. Estudantes de Odontologia.

\section{INTRODUÇÃO}

Há muito se vem observando a falência do paradigma curativista na área biomédica, refletindo no processo de formação de profissionais que atuam nessa área. Portanto, tornou-se essencial a mudança de cenários nos quais se realiza o processo ensinoaprendizagem para locais mais representativos da realidade sanitária e social brasileira, integrando serviço e pesquisa, visando a uma abordagem problematizadora, ao compromisso ético, humanístico e social ${ }^{1}$.

Tais considerações vêm ao encontro das diretrizes curriculares propostas para o curso de Odontologia, aprovadas pela Resolução CNE/CES no 3/2002², que dizem: "o Curso de Graduação em Odontologia tem como perfil do formando egresso/ profissional o Cirurgião-Dentista capacitado ao exercício de atividades referentes à saúde bucal da população, pautado em princípios éticos, legais e na compreensão da realidade social, cultural e econômica do seu meio, dirigindo sua atuação para a transformação da realidade em beneficio da sociedade."

Uma das principais ferramentas da promoção de saúde é a educação, na medida em que proporciona aos indivíduos o resgate de sua autonomia, a percepção de valores e conhecimentos, o desenvolvimento de uma visão crítica e do empoderamento da população.
De acordo com L'Abbate ${ }^{3}$, todo profissional de saúde é um educador em saúde em potencial. É condição essencial para a sua prática o seu próprio reconhecimento como sujeito do processo educativo, evidenciando a relevância da formação desses profissionais com essa visão.

O objetivo desta pesquisa foi analisar a percepção dos acadêmicos do Curso de Odontologia sobre o tema "Educação em Saúde Bucal", bem como investigar o valor e a importância que eles atribuem a essa prática desenvolvida em atividade curricular em outros cenários.

\section{MATERIAIS E MÉTODOS}

A pesquisa, caracterizada como um estudo exploratório descritivo, com abordagem quantiqualitativa, foi aprovada pelo Comitê de Ética em Pesquisa da Faculdade de Odontologia de Araçatuba, Universidade Estadual Paulista, Brasil (Processo FOA, $\left.{ }^{\circ} 01843,2007\right)$.

Foi pesquisado o universo dos pesquisados de acadêmicos $(\mathrm{n}=78)$ matriculados no último ano do curso integral de Odontologia da FOA - Unesp, no ano letivo de 2009. O grupo de acadêmicos que integra a pesquisa foi organizado com base em dois critérios: estar cursando o último ano de graduação em Odontologia e aceitar participar da pesquisa.

No último ano do curso, a disciplina

\footnotetext{
${ }^{1}$ Departamento de Medicina Social, Centro de Ciências da Saúde, Universidade Federal do Espírito Santo (UFES), Vitória, ES, Brasil ${ }^{2}$ Departamento de Odontologia Infantil e Social, Faculdade de Odontologia de Araçatuba, Universidade Estadual Paulista "Júlio de Mesquita Filho" (UNESP), Araçatuba, SP, Brasil

Contato: kktonini@yahoo.com.br, nino_redacao@yahoo.com.br, cgarbin@foa.unesp.br
} 
Odontologia Preventiva e Sanitária propõe atividades em outros cenários de práticas. Uma delas é realizada em Escolas Municipais de Educação Infantil. Os pré-escolares, então, constituem o grupo-alvo de um Programa de Educação em Saúde Bucal, que tem como objetivo proporcionar o desenvolvimento de suas capacidades cognitivas, afetivas e psicomotoras, relativas à saúde bucal. Essa iniciativa foi desenvolvida a partir da necessidade de disseminar os saberes odontológicos para o universo infantil, com a finalidade de promover saúde e melhorar os níveis de saúde bucal.

Durante as aulas teóricas da disciplina, são discutidos a forma de abordagem da criança, a linguagem adequada para cada faixa etária, os instrumentos (canal de comunicação) e os temas que serão focalizados em campo. Didaticamente, no trabalho com as crianças, os acadêmicos lançam mão das dramatizações, de desenhos e pinturas, da música, do faz de conta, dos meios audiovisuais e das atividades ludopedagógicas. Todo material didático utilizado é produzido pelo próprio acadêmico.

Os sujeitos da pesquisa foram esclarecidos quanto aos objetivos. Aqueles que consentiram a participação tiveram suas identidades resguardadas em cumprimento aos preceitos éticos contidos na Resolução n 196/96 do CNS/MS.

Os dados foram coletados por meio de questionário semiestruturado, autoaplicado, com perguntas abertas e fechadas, especialmente elaborado para a pesquisa. As questões propostas foram: o que você entende por Educação em Saúde Bucal? Você acha importante a realização de Programas de Educação em Saúde Bucal nas escolas? Em sua opinião, os Programas de Educação em Saúde bucal nas escolas são eficazes? Você acredita que a sua participação nessa atividade, durante a sua graduação, impacta de alguma maneira sua vida?

Para a análise qualitativa, utilizou-se a análise de conteúdo, por meio da técnica de análise de categoria ${ }^{4}$, formada a partir das respostas dos sujeitos da pesquisa. Foram seguidos, ainda, os princípios propostos por Minayo ${ }^{5}$, os quais visam ao agrupamento de circunstâncias que dão sentido ao fato, e não a uma situação isolada, oferecendo liberdade para resgatar o que for importante na novidade dos temas, mesmo se a frequência for pequena.

No processamento dos dados quantitativos e análise estatística descritiva, foi empregado o software de domínio público EPI INFO3.2.

\section{RESULTADOS}

Do total de 78 acadêmicos, $69,2 \% \quad(n=54)$ consentiram em participar do estudo. Desses, $66,7 \%$ pertenciam ao sexo feminino. Noventa e oito por cento dos acadêmicos acreditam que a sua participação nas atividades de educação em saúde bucal tiveram impacto na vida acadêmica e pessoal. Cem por cento dos alunos relataram achar importante a realização de Programas de Educação em Saúde Bucal nas escolas, e 74\% acreditam na eficácia desses programas.

Em relação às respostas positivas dadas pelos acadêmicos sobre o porquê de os Programas de Educação em Saúde Bucal nas escolas serem eficazes, as seguintes categorias foram formadas:

- Categoria 1 - Aquisição ou mudanças de hábitos desde cedo: "Porque, em muitos casos, a criança transmite em sua casa o que aprendeu na escola e muda seus hábitos e os de sua família".

- Categoria 2 - Facilidade de aprendizado nessa idade: "Porque, nessa fase, as crianças estão dispostas a aprender e, conseqüentemente, levam para a casa o conhecimento obtido".

- Categoria 3 - Motivação em frente ao aprendizado: "Porque, só a partir do momento que a criança entende o porquê da realização de medidas preventivas, ela se sente motivada a realizá-las".

- Categoria 4 - Desconhecimento prévio das crianças: "Muitas crianças não têm noção de higiene em casa, assim, nas escolas, elas aprendem o correto e levam o conhecimento para casa demonstrando para os pais e familiares".

- Categoria 5 - A escola como um ambiente de aprendizado: "Porque a escola já é um ambiente de ensino e deve o ser para qualquer assunto, assim como o de saúde".

As categorias descritas abaixo são referentes ao entendimento dos acadêmicos sobre Educação em Saúde Bucal:

- Categoria 1 - Ensino de métodos de higienização e controle da dieta: "Consiste em ensinar as pessoas a escovar os dentes de maneira correta, a criar o hábito de passar fio dental e. conforme as condições sócioeconômicas, visitar o cirurgião-dentista a cada seis meses".

- Categoria 2 - Transmissão de informações sobre saúde bucal: "Passar para as crianças e adultos a importância da saúde bucal, ensinando como manter essa saúde que é tão importante".

- Categoria 3 - Conscientização sobre a importância da saúde bucal: "Um trabalho preventivo, que busca a conscientizar as pessoas da necessidade de cuidar da higiene bucal, de evitar doenças e fazê-las entender a relação da saúde bucal com a saúde sistêmica".

- Categoria 4 - Instrumento de prevenção de doenças bucais: "Essa educação tem por objetivo ensinar a um determinado grupo como prevenir $e$ cuidar de sua saúde bucal".

- Categoria 5 - Conhecimento adquirido ao longo da vida: "Compreende os conhecimentos adquiridos ao decorrer da vida que impedem o 
desenvolvimento de microrganismos presentes na cavidade bucal por meio de escovação e fio dental".

- Categoria 6 - Conjunto de atividades que visam a melhorar a saúde bucal da população: "Ações no âmbito coletivo que têm como meta a melhoria da saúde bucal da população”.

- Categoria 7 - Um programa: "Eu entendo como um programa, um projeto cujo objetivo busca ensinar as pessoas e esclarecer questões sobre saúde bucal”.

\section{DISCUSSÃO}

Nesta pesquisa, 69,2\% dos acadêmicos aceitaram participar do estudo. Essa porcentagem é semelhante a do estudo conduzido por Bhayat et al. ${ }^{6}$, realizado também com estudantes de Odontologia, que foi de 64,0\%. Já Mialhe e Silva ${ }^{7}$, analisando a percepção de estudantes de Odontologia sobre Educação em Saúde, identificaram uma maior porcentagem, $85,3 \%$ da população-alvo.

Da mesma forma que neste estudo, Gontijo et al. ${ }^{8}$, analisando a saúde bucal coletiva na visão do estudante de Odontologia, constataram que a maioria dos respondentes era do sexo feminino. Esse fator corrobora a chamada "feminização da Odontologia", 10 , uma tendência de todos os cursos de nível superior.

A maioria dos graduandos acredita que a sua participação nas atividades em outros cenários de práticas - de Educação em Saúde Bucal que realizam nas escolas - durante a graduação, teve impacto na vida acadêmica e pessoal, uma vez que puderam entrar em contato com novas e diferentes realidades.

Gontijo et $\mathrm{al}^{8}{ }^{8}$ obtiveram um índice de aprovação de graduandos em Odontologia de $92 \%$, referente a uma atividade extramuro, porém somente $8 \%$ de respostas consideraram que não houve nenhuma contribuição para sua formação. Saliba et al. ${ }^{11}$ acreditam que experiências como essas são extremamente válidas, pois, fora do ambiente acadêmico tradicional, o aluno tem oportunidade de desenvolver sensibilidade e responsabilidade social, por meio de uma participação mais ativa na comunidade.

Medeiros Júnior et al. ${ }^{12}$, em seu estudo, puderam verificar que a atividade desenvolvida em um hospital público trouxe a oportunidade de interação do aluno de Odontologia com outras profissões da saúde, crescimento individual e coletivo e também a vivência de experiências diferentes e enriquecedoras do ponto de vista da formação humana e profissional, uma vez que trabalhavam com saúde bucal, sem perder a visão do paciente como um todo.

Nesta pesquisa, todos acham importante a realização de Programas de Educação em Saúde Bucal nas escolas e a maioria acredita na eficácia desses programas. Foi relatada e comprovada por diversos autores a importância dos Programas de Educação em Saúde nas escolas de educação infantil, tanto na aquisição de novos conhecimentos, como na redução dos índices das doenças bucais ${ }^{13-17}$.

De acordo com a Organização Mundial da Saúde $^{18}$, as escolas são consideradas ótimos espaços para serem realizadas ações de Educação em Saúde Bucal, e os estudantes podem ser acessados durante todos os anos de sua formação, desde a infância até a adolescência. Esses estágios são importantes na vida dos alunos, pois os comportamentos relacionados com a saúde bucal, bem como crenças e atitudes estão sendo desenvolvidos.

As falas dos acadêmicos foram unânimes em relação à facilidade que a criança apresenta de mudar hábitos errôneos e de aprendizagem. Kwan et al. ${ }^{19}$ relataram que a criança tem condições de aplicar, em seu cotidiano, a experiência vivenciada na escola e pode agir como agente multiplicador de saúde dentro do ambiente familiar.

Garbin et al. ${ }^{20}$ constataram, em sua pesquisa, por meio da percepção de pais de pré-escolares, que as crianças participantes de um Programa de Educação em Saúde Bucal foram capazes de levar o conhecimento adquirido na escola para seus familiares, inclusive mudando a rotina de saúde bucal da sua família.

Para Valla e Stotz ${ }^{21}$, é importante estar atento ao fato de que toda criança chega à escola com um saber. De acordo com os autores, isso não pode ser ignorado, já que todas as pessoas acumulam experiências, valores, crenças e conhecimentos. Muitas vezes, o profissional de saúde apresenta o saber científico, mas desconsidera o saber da população, posto como inferior.

Algumas falas, como "Nas escolas elas aprendem o correto"; "Muitas crianças chegam na escola sem saber como cuidar e prevenir doenças"; "Falta de informação das crianças"; "Muitas daquelas crianças não têm educação doméstica necessária para prevenir enfermidades"; "Induzem crianças que não tem noção nenhuma sobre noções de saúde bucal", deixam claro que os acadêmicos podem estar desconsiderando esse conhecimento prévio.

Outro ponto relevante foi a percepção de um acadêmico sobre a escola ser um ambiente de ensino para qualquer tema, corroborando a literatura, que enfatiza o fato de ela ser um ambiente propício para a aplicação de Programas de Educação em Saúde, pois está inserida em todas as dimensões do aprendizado ${ }^{22}$.

Do mesmo modo que a idade pré-escolar foi citada como uma das causas de eficácia de Programas de Educação em Saúde Bucal, devido à facilidade de aprendizado, ela apareceu como fator de não eficácia, 
na opinião de alguns acadêmicos que relataram que, nessa idade, as crianças não são capazes de aprender: "Deveria ser realizado com crianças entre 9-12 anos para que elas possam entender com maior facilidade o que queremos passar"; "Porque, na maioria das vezes, atingimos crianças muito novas que não possuem capacidade de entender o que é ensinado, devendo, então, ter um contato mais direto com os pais e professores"; "Acredito que, em certas faixas etárias, eles sejam pouco eficazes".

Para Bijella et al. ${ }^{23}$, a idade pré-escolar, ou seja, a primeira infância, constitui-se um grupo prioritário de trabalho, especialmente na Odontologia, pois, além dessas crianças apresentarem alto risco da doença cárie, elas possuem grande capacidade de imitar ações que presenciem e apresentam facilidade em alterar hábitos errôneos.

Quando os acadêmicos foram indagados a respeito do seu entendimento sobre Educação em Saúde Bucal, percebeu-se, em suas respostas, uma visão reducionista. Mialhe e Silva ${ }^{7}$, da mesma forma, observaram, em sua pesquisa, que o conceito de Educação em Saúde dos acadêmicos de Odontologia avaliados estava fortemente ligado ao conceito positivista de ensinamento, instrução e prevenção de doenças, pautado em orientações e informações transmitidas à comunidade pelos profissionais.

Silveira et al. ${ }^{24}$ ressaltaram a importância de que programas educativos em saúde bucal não devem ser limitados ao trinômio placa-dieta-escovação e à transferência de informações sanitárias ou ainda restritos à preconização de hábitos saudáveis de autocuidados.

Pauleto et al. $^{25}$ relataram que, apesar da existência de vários programas, a dimensão educativa é pouco desenvolvida e, quando realizada, está fortemente apoiada em práticas de transmissão de conhecimentos, sem espaço para práticas dialógicas capazes de mobilizar as crianças quanto à problemática da saúde bucal, que visa à autonomia em relação ao cuidado com a saúde.

Guterman $^{26}$, estudando a percepção de graduandos sobre o tema Educação em Saúde, averiguou que os temas mais citados foram "prevenção" e "educação", aludindo que eles não conseguiram transpor a barreira do paradigma preventivista para as ações educativas.

De acordo com a Fundação Nacional de Saúde ${ }^{27}$, a prática educativa em saúde deve ser vista de maneira ampla, e não como uma mera relação de ensino/aprendizagem didatizada e assimétrica, mas sim como uma relação dialógica pautada na horizontalidade entre os seus sujeitos, como atribuição de todo trabalhador de saúde.

Essa concepção reducionista dos graduandos, centrada na doença, pode ser consequência de sua própria formação acadêmica, que pode estar sendo pautada em uma abordagem curativista e tecnicista, em que nem todas as disciplinas do curso atribuem o mesmo valor à Educação em Saúde Bucal.

Gontijo et al. ${ }^{8}$ ressaltaram a importância de os docentes utilizarem metodologias educacionais que contribuam para a formação acadêmica. Como bem comenta Araújo ${ }^{28}$, “[...] esse processo deve ultrapassar o campo da saúde bucal coletiva, estendendo-se para todas as áreas, acabando com as dicotomias entre básico e clínico, entre clínico e social e entre público e acadêmico".

\section{CONCLUSÃO}

Os resultados deste estudo permitem concluir que:

a) a maioria dos acadêmicos relatou que sua participação nas atividades em outros cenários de práticas trouxe impacto na vida acadêmica e pessoal;

b) os acadêmicos reconhecem a importância dos Programas de Educação em Saúde Bucal no âmbito escolar;

c) o conceito de "Educação em Saúde Bucal" precisa ser trabalhado melhor, uma vez que o entendimento reducionista dos alunos sobre o referido conceito pode prejudicar a atuação deles com as crianças e influenciar os objetivos do programa.

\section{ABSTRACT}

Aim: To analyze dental students' view concerning Oral Health Education. Materials and Methods: The present research, using both qualitative and quantitative approaches, addresses aspects related to dental students' understanding of Oral Health Education, the value that they attach to this practice in the school environment, as well as the importance of their participation in these and other activities during their studies in dentistry. Results: Although dental students recognize the importance of Oral Health Education in the school environment,as well as the importance of their own participation within these activities, their perception of "Oral Health Education" is reductionist and focused primarily on the disease. Conclusion: It is well-known that these findings are reflections and consequences of an established context within a university education. It is therefore necessary that these professional educators perform an in-depth rethinking of the dental practice, in an attempt to develop methodologies based on Health Education in their own teaching practices.

Uniterms: Health education. Dentistry. Oral health. Dental students.

\section{REFERÊNCIAS}

1. Campos FE, Belisário AS. O programa da Saúde da 
Família e os desafios para a formação profissional e a educação continuada. Interface Comuni Saúde Educ. 2001; 5:133-42.

2. Ministério da Saúde. Conselho Nacional de Educação. Câmara de Educação Superior. Resolução $\mathrm{n}^{\circ} 3$ de 19 de fevereiro de 2002. Institui Diretrizes Curriculares Nacionais do Curso de Graduação em Odontologia. Diário Oficial da República Federativa do Brasil, 2002.

3. L'abbate S. Educação em saúde: uma nova abordagem. Cad Saúde Pública. 1994; 10:481-90.

4. Bardin L. Análise de conteúdo. Lisboa: Edições 70; 1994.

5. Minayo MCS. O desafio do conhecimento: pesquisa qualitativa em saúde. $8^{a}$ ed. São Paulo: Hucitec. 2004.

6. Bhayat A, Yengopal V, Rudolph MJ, Govender U. Attitudes of south african dental therapy students toward compulsory community service. J Dent Educ. 2008; 72:1135-41.

7. Mialhe FL, Silva CMC. A educação em saúde e suas representações entre alunos de um curso de Odontologia. Ciênc Saúde Coletiva. 2011; 16 suppl. 1:155-61.

8. Gontijo LPT, Almeida MCP, Gomide LRS, Barra RP. A saúde bucal coletiva na visão do estudante de odontologia: análise de uma experiência. Ciênc Saúde Coletiva. 2009; 14:1277-85.

9. Moimaz, SAS, Saliba NA, Blanco MRB. A força do trabalho feminino na odontologia, em Araçatuba, SP. J Appl Oral Sci. 2003; 11:301-5.

10. Costa SM, Durães SJA, Abreu MHNG. Feminização do curso de odontologia da Universidade Estadual de Montes Claros. Ciênc Saúde Coletiva. 2010; 15:1865-73.

11. Saliba NA, Pereira AA, Moimaz SAS, Garbin CAS, Arcieri RM. Programa de educação em saúde bucal: a experiência da Faculdade de Odontologia de Araçatuba, UNESP. Odontol Clín-Científ. $2003 ; 2: 197-200$

12. Medeiros Júnior A, Alves MSCF, Nunes JP, Costa ICC. Experiência extramural em hospital público e a promoção da saúde bucal coletiva. Rev Saúde Pública. 2005; 39:305-10.

13. Ohara S, Kawaguchi Y, Shinada K, Sasaki Y. Evaluation of school-based dental health activities including fluoride mouth-rinsing in Hiraizumi, Japan. J Med Dent Sci. 2000; 47:133-41.
14. Mastrantonio SDS, Garcia PPNS. Programas educativos em saúde bucal: revisäo da literatura. JBP, J Bras Odontopediatr Odontol Bebê. 2002; $5: 215-22$.

15. Pereira AP. Avaliação do programa de educação em saúde bucal da Faculdade de Odontologia de Araçatuba. [dissertação]. Araçatuba (SP): Faculdade de Odontologia de Araçatuba da Universidade Estadual Paulista; 2002.

16. Aquilante AG, Almeida BS, Castro RFM, Xavier CRG, Peres SHCS, Bastos JRM. A importância da educação em saúde bucal para pré-escolares. Rev Fac Odontol UNESP. 2003; 32:39-45.

17. Goel P, Sehgal M, Mittal R. Evaluating the effectiveness of school-based dental health education program among children of different socioeconomic groups. J Indian Soc Pedod Prev Dent. 2005; 23:131-3.

18. World Health Organization. The World Oral Health Report 2003. Continuous improvement of oral health in the 21st century: the approach of the WHO Global Oral Health Programme. Geneva: World Health Organization; 2003.

19. Kwan SL, Petersen EP, Pine CM, Annerose B. Health-promoting schools: an opportunity for oral health promotion. Bull World Health Organ. $2005 ; 83: 677-85$.

20. Garbin CAS, Garbin AJI, Santos KT, Pereira DL. Oral health in schools: promoting health agents. Int J Dent Hyg. 2009; 7:212-5

21. Valla V, Stotz E, organizadores. Participação popular, educação e saúde: teoria e prática. $2^{\mathrm{a}}$ ed. Rio de Janeiro: Relume-Dumará; 1993.

22. Fernandes MH, Rocha VM, Souza DB. A concepção sobre saúde do escolar entre professores do ensino fundamental ( $1^{\mathrm{a}}$ a $4^{\mathrm{a}}$ séries). Hist Ciênc Saúde. 2005; 12:283-91.

23. Bijella MFTB, Bijella VT, Figueiredo MC. Avaliação de um programa odontológico, com bases educativa, preventiva e curativa, desenvolvido com pré-escolares durante 12 meses. Cecade News. 1995;3:1-5.

24. Silveira JLGC, Campos ML, Berndt RLE. Educação em saúde como estratégia para o controle social em saúde bucal. Pesqui Bras Odontopediatria Clín Integr. 2006; 6:29-34.

25. Pauleto ARC, Pereira MLT, Cyrino EG. Saúde bucal: uma revisão crítica sobre programações educativas para escolares. Ciênc Saúde Coletiva. 
2004; 9:121-30.

26. Guterman N. O cirurgião-dentista como educador em saúde bucal: explorações em torno de uma prática. Rev ABENO. 2005; 5:115-24

27. Fundação Nacional de Saúde. Diretrizes de educação em saúde visando à promoção da saúde: documento base: documento I/ Fundação Nacional de Saúde. Brasília: Funasa; 2007.

28. Araújo ME. Palavras e silêncios na educação superior em odontologia. Ciênc Saúde Coletiva.2006; 11:179-82.

Recebido em 26/10/2011 - Aceito em 15/02/2012

\section{Autor correspondente:}

Cléa Adas Saliba Garbin

UNESP - Faculdade de Odontologia - Odontologia Preventiva e Social

Rua José Bonifácio, 1193 - Vila Mendonça

CEP: 16015-050 - Araçatuba - SP - Brasil

Email: cgarbin@foa.unesp.br 